\title{
Self-Deployable Joints for Ultra-Light Space Structures
}

\author{
S. Ferraro* and S. Pellegrino* \\ California Institute of Technology, Pasadena, CA 91125
}

\begin{abstract}
The paper presents ongoing research and development of novel concepts for deployable space structures using self-latching, flexural joints to replace mechanical hinges. The mechanics of deformation of Fiber-Reinforced-Polymers (FRP) joints for in-plane deployment mechanisms are studied. Methods for characterizing these joints via experiments and numerical simulations are proposed. A failure criterion suitable for ultra-thin, plain-weave composites is used to predict failure of the joints and achieve a successful design.
\end{abstract}

\section{Nomenclature}

$\begin{array}{ll}E_{1 f}, E_{2 f} & \text { Fibers Young's moduli } \\ E_{m} & \text { Matrix Young's modulus } \\ F_{1 t}, F_{2 t} & \text { Tensile strength parameters } \\ F_{1 c}, F_{2 c} & \text { Compressive strength parameters } \\ F_{3} & \text { In-plane shear strength parameter } \\ F_{4} & \text { Bending strength parameter } \\ F_{6} & \text { Twisting strength parameter } \\ F I_{1} & \text { In-plane failure index } \\ F I_{2} & \text { Bending failure index } \\ F I_{3} & \text { Coupled in-plane and bending failure index } \\ f_{i}, f_{i j} & \text { Failure coefficients, combination of strength parameters } \\ G & \text { Composite shear modulus } \\ G_{m} & \text { Matrix shear modulus } \\ K_{B} & \text { Joint bending stiffness } \\ M_{x}, M_{y}, M_{x y} & \text { Bending and twisting moments on a flat plate } \\ N_{x}, N_{y}, N_{x y} & \text { In-plane and shear stress resultants on a flat plate } \\ V_{f} & \text { Fiber volume fraction } \\ \gamma_{y} & \text { In-plane yield shear strain } \\ \theta_{1}, \theta_{2} & \text { folding angles } \\ \tau_{y} & \text { In-plane yield shear stress } \\ \varphi_{0} & \text { Fiber misalignment angle }\end{array}$

\section{Introduction}

Traditional concepts for deployable space structures use kinematic solutions based on mechanism theory ${ }^{1}$ in order to package the structure during launch and deploy it into its operational configuration in space. Mechanical hinges can be replaced with lighter, smaller and cheaper continuous elements made from ultrathin composites. Solutions that promote the concept of lightweight joints have already been proposed. Two examples are tape-spring rolamite (TSR) hinges ${ }^{2}$ and compliant hinges. ${ }^{3}$ TSR hinges are composed of two metal measuring tape sections and two sets of cams constrained to roll on each other. The deployment

\footnotetext{
*PhD candidate, Graduate Aerospace Laboratories, 1200 East California Boulevard, Mail Code 205-45. e-mail: sferraro@caltech.edu

$\dagger$ Joyce and Kent Kresa Professor of Aeronautics and Civil Engineering, Graduate Aerospace Laboratories, 1200 East California Boulevard, Mail Code 105-05, AIAA Fellow. e-mail: sergiop@caltech.edu
} 
kinematics are controlled by the rolling cams and elastic latching into the deployed configuration is provided by tape springs. This joint achieves highly repeatable and accurate positioning but can be improved in terms of volume and weight. Compliant hinges made of carbon fiber composites provide a much lighter solution but require precision assembly of the structure, composed of hinges and connecting struts, to achieve a stress free state of the deployed structure. Internal assembly loads would result in deformation of the compliant hinges and loss of shape accuracy. Hinges incorporating elastic memory composites have also been shown to be a viable approach for lightweight, cost-effective mechanisms, providing controlled, low-shock deployment and structural efficiency. ${ }^{4,5}$

Recent studies have proposed building foldable, modular units ${ }^{6,7}$ for in-space assembly of telescope backplane structures that support large-aperture primary mirrors. One of the cornerstones of this idea is the use of identical segments and modules to build the primary mirror. An example is shown in Figures 1(a) and 1(b). Among the benefits of this concept, the two most important are: first, cutting the cost of manufacturing by avoiding specialized and single-use parts, and second, robustness to possible failures during assembly and operation by launching a small number of spare parts to replace any damaged or malfunctioning segments. In contrast, a primary mirror built with uniquely shaped segments would require a large number of spare parts, which would increase cost and decrease mass and volume efficiency. Modular and self-deployable structures can not only enable extremely large space telescopes, lowering their cost, but also extend the capability of an already working system by implementing the concept of an Evolvable Space Telescope. ${ }^{8}$

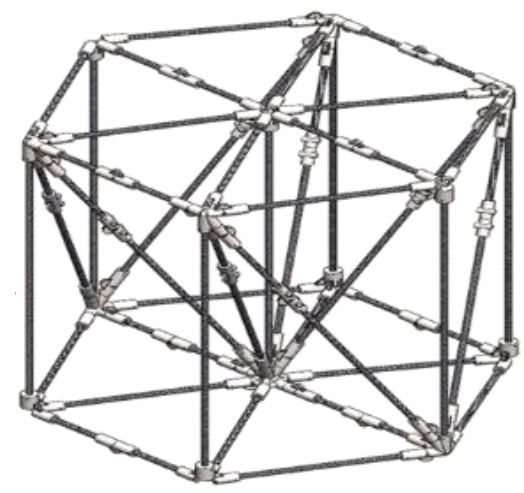

(a)

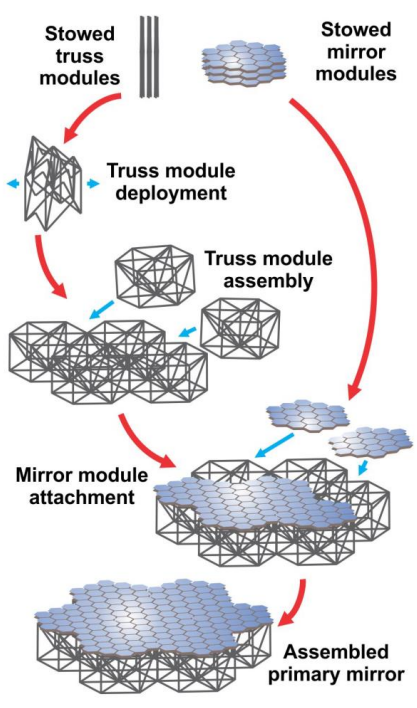

(b)

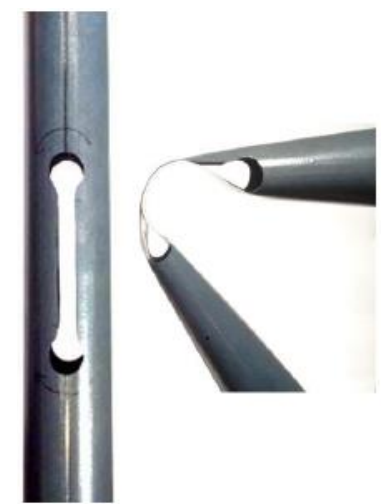

(c)

Figure 1. (a) self-deployable truss module concept, ${ }^{6}$ (b) telescope primary mirror assembly concept, ${ }^{7}$ (c) example of tape spring hinge.

The simple and low-cost concept of ultra-thin tape-spring hinges shown in Figure 1(c), made by cutting small slots in a thin-walled tube, ${ }^{9}$ can be extended to build a compact module, made entirely of fiberreinforced polymers, which can reliably self-deploy using the potential energy of its stowed configuration. The main advantage of this approach is the implementation of a lightweight technology that can provide low-cost building blocks for large space structures. Tape-spring hinges are traditionally built in straight tubes. Foldable corner joints that use the idea of tape-spring hinges have not been studied before. The module shown in Figure 1(a) relies on TSR hinges to fold and self-deploy. Progress towards composite, flexural joints must be made to replace heavier kinematic hinges and achieve a new type of self-deployable module built uniquely from composite laminates.

The present paper focuses on a novel concept for self-deployable joints, looking at different designs entirely built with Fiber-Reinforced-Polymers (FRP). First, different iterations of FRP joints are introduced and the challenges in folding initial prototypes are explained through an insight into their deformation mechanisms. Next, the joints are characterized via experiments, a failure criterion, and numerical simulations. The results lead to an improved design for self-deployable joints. Finally, a silicone-molding manufacturing technique is presented. 


\section{Fiber-Reinforced-Polymers (FRP) Self-Deployable Joints}

Fiber-Reinforced-Polymers (FRP), self-deployable joints for in-plane deployment mechanisms were studied. A miter joint shape, where two thin-walled cylinders intersect at 90 degrees, was chosen to avoid complex folding mechanisms, thus enabling a study more focused on the mechanics of deformation of the joint as it folds. This simpler shape is an initial step towards designing the kind of complex corner joints in Figure 1(a). The objective of this study was to achieve a fully folded configuration of the joint. This is achieved when the two tubes rotate 40 degrees each in order to come into contact in the folded configuration. The total folding angle, $\theta$, is the sum of the two folding angles, $\theta_{1}$ and $\theta_{2}$, shown in Figure 2. The tubes come into contact when $\theta_{1}=\theta_{2}=40$ degrees and not 45 degrees because of their diameter. In this study, the joints were not designed to allow the tubes to flatten. Four requirements were set for the joints. They must be stiff when deployed, compliant during folding (to avoid failure), ultra-lightweight, and self-deployable.

\section{A. Rigid Molding Manufacturing}

Initial prototypes of the joints were made with plain-weave 525 Astroquartz II fabric (quartz fiber), from JPS Composite Materials, pre-impregnated with PMT-F4 epoxy resin, from Patz Materials \& Technologies. The fibers Young's modulus is $E_{1 f}=E_{2 f}=72 \mathrm{GPa}$. Quartz fiber composites were chosen because of their higher ultimate strain, measured at $2.6 \%$, compared to carbon fiber composites. Each ply has a measured thickness of $90 \mu \mathrm{m}$ and areal density of $105 \mathrm{gsm}$. First, three prototypes were built from two separate tubes with circular cross section, $31.75 \mathrm{~mm}$ in diameter. A 45 degree angle cut was made at the end of the tubes that were subsequently joined with epoxy coating and cured at room temperature. These prototypes displayed a weak bond at the intersection due to the brittleness of the epoxy layer, which failed when the joints were loaded by the folding process. As a consequence, it was decided to co-cure the two tubes as a single part.

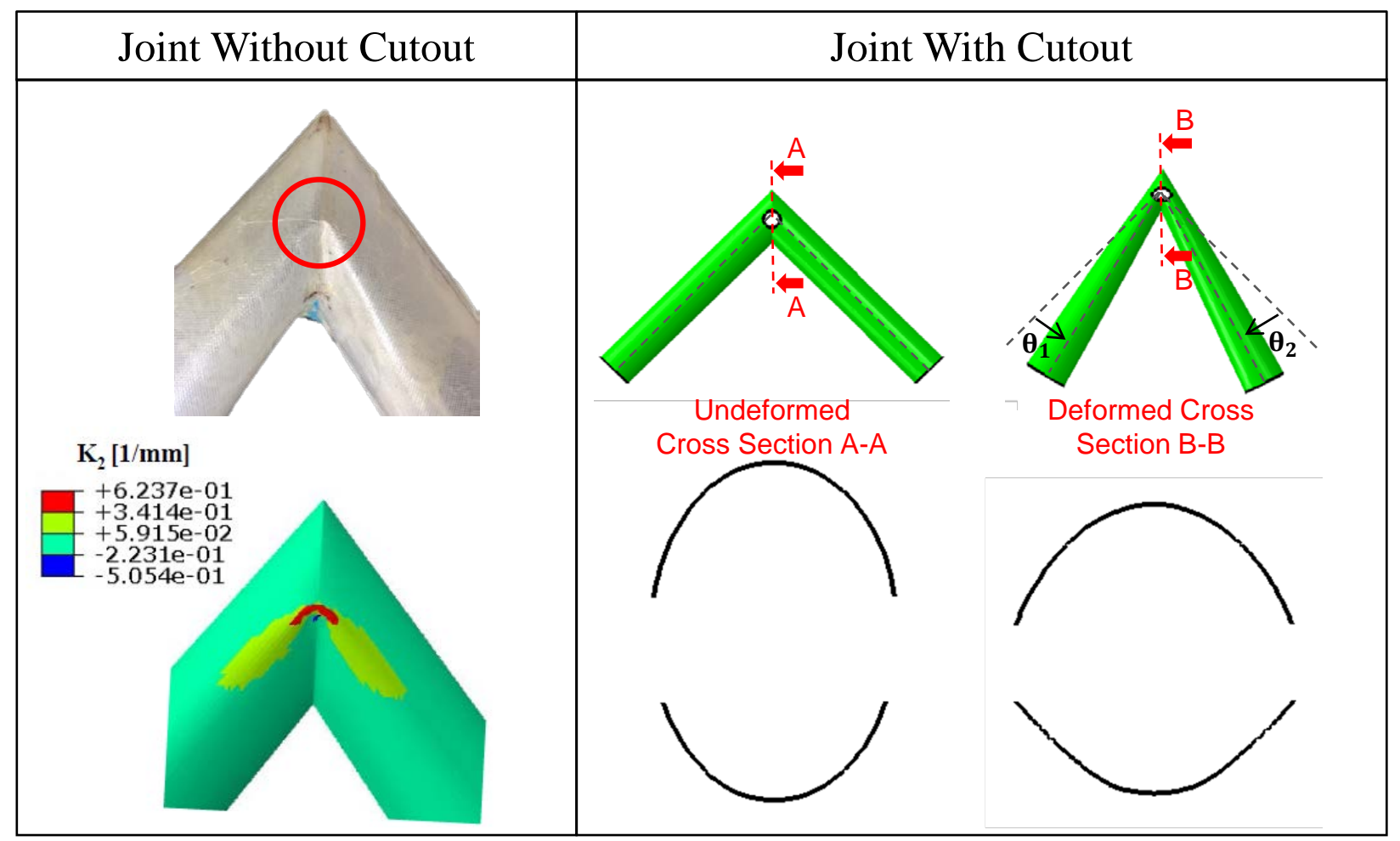

Figure 2. On the left, a physical model and numerical simulation of FRP joint without cutouts and partially folded. The contour plots on the simulation show the change in circumferential curvature of the partially folded joint. On the right, shape of undeformed and deformed cross sections of FRP joint, with circular cutout, obtained from numerical simulations. 
The manufacturing procedure adopted at this step was designed to overcome the complications of the non-zero Gaussian curvature at the joint between the two cylindrical surfaces. An aluminum mold made of three components, two cylindrical shafts (length $230 \mathrm{~mm}$, diameter $31.8 \mathrm{~mm}$ ) and one long screw to connect both, was used. One ply of the layup was cut into four geometric patterns and assembled directly on the mold, thus creating overlapping strips of material along the axial direction of each shaft. If the layup consisted of more than a single ply, the subsequent plies were assembled following an identical procedure. The layup was wrapped in a release layer and high-temperature polyolefin shrinking tubes, to reduce wrinkles, followed by a commonly used vacuum bagging application. After curing in the autoclave, the screw allowed the two aluminum shafts to be separated and extracted from the composite part, which remained intact.

The first iteration of the co-cured joint is shown in Figure 2. Considering that the 0 degrees direction of the fibers corresponds to the axial direction of each tube, the layup is composed of a single, plain-weave ply oriented at \pm 45 degrees. As a result of the manufacturing procedure, four regions of overlapping plies, and hence having double thickness, run along the axial direction of the tubes. The two-ply regions are 5 $\mathrm{mm}$ wide with layup [45/45], where 45 indicates a plain weave lamina consisting of fibers oriented at \pm 45 degrees. These regions increased the stiffness of the joint. Figure 3 helps visualizing the overlapping regions of the third iteration FRP joints.

Preliminary folding experiments were performed. The experiments showed that when the joint is folded, the deformation along the tubes transitions from a longitudinal ovalization of the cross section at the base, if the base is left free to ovalize as explained in section IV, to a transverse flattening of the cross section at the joint between the two cylindrical surfaces. Figure 2 shows the flattening behavior. The length of the tubes was chosen, from experimental observations, to position the joint between the two cylindrical surfaces far enough from the base of each tube. This allows for a transition region and reduces stress in the joint. The flattening of the cross section at the joint between the two cylindrical surfaces creates regions of localized curvature, which were observed during experiments and simulated numerically. More details about numerical simulations and folding experiments are discussed later in this paper. Figure 2 shows how cutouts can eliminate the regions of localized curvature. Nevertheless, they reduce the overall stiffness of the joint.

To address both stiffness and folding requirements, the designed of the joint was further evolved. Circular cutouts with diameter $\mathrm{D}=14 \mathrm{~mm}$ were placed at the intersection. They enabled the joint to fold up to 17 degrees without any sign of failure. The layup was also changed to a three-ply configuration $[45 / 0 / 45]$, where 0 indicates a plain weave lamina consisting of fibers oriented at 0 and 90 degrees. In this case, the areas of double thickness had six plies oriented as follows [45/45/0/0/45/45]. The matrix was also changed from epoxy to PMT-F6 cyanate ester resin, from Patz Materials \& Technologies, to address future concerns regarding outgassing in space. The matrix modulus is $E_{m}=3.64 \mathrm{GPa}$.

A third iteration of the FRP joint enabled a higher folding angle. The layup, shown in Figure 3, consists of four different regions of two, three, four and six plies respectively. The plies are oriented as follows: two-ply regions [45/45], three-ply regions [45/0/45], four-ply regions [45/45/45/45], and six-ply regions $[45 / 45 / 0 / 0 / 45 / 45]$.
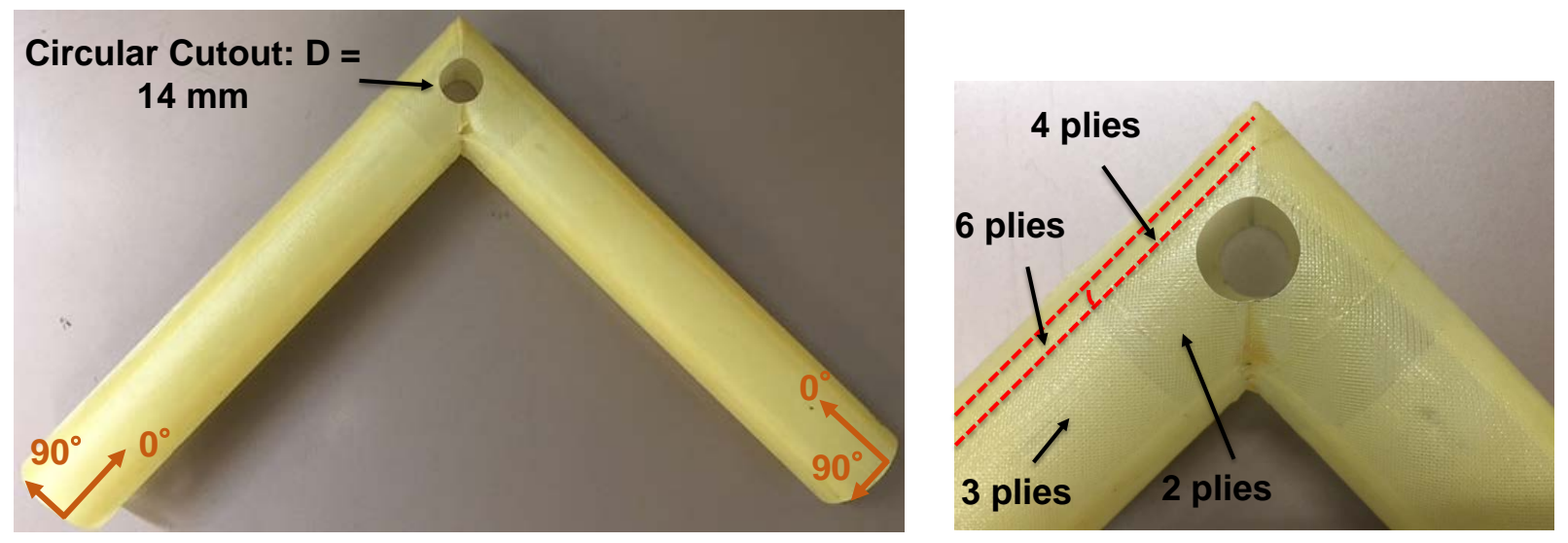

Figure 3. Third iteration prototype. 


\section{B. Folding Experiments}

Folding experiments were performed on four of the prototypes described in the previous section and reaction moments from the two tubes of each joint were recorded and plotted against the folding angle. The momentangle curves are shown in Figure 4(b). The orange and red curves were obtained from tests performed on second iteration prototypes, with three plies at the intersection. The average bending stiffness of these joints, obtained from the initial, linear part of the experimental moment-angle curves, was measured at $K_{B}$ $=4193.7[\mathrm{~N} \mathrm{~mm}]$. The joints cannot fold more than 17 degrees before failure occurs. Failure was defined as a large crack, $5 \mathrm{~mm}$ or longer, appearing near the cutout. The green and blue curves were obtained from tests performed on third iteration prototypes, with two plies at the intersection. The average bending stiffness was measured at $K_{B}=1951.9$ [N mm]. While delamination or small cracks, shorter than $1 \mathrm{~mm}$, were already visible around the cutout at 15 degrees of folding angle, the joints could be folded up to 50 degrees without failing.

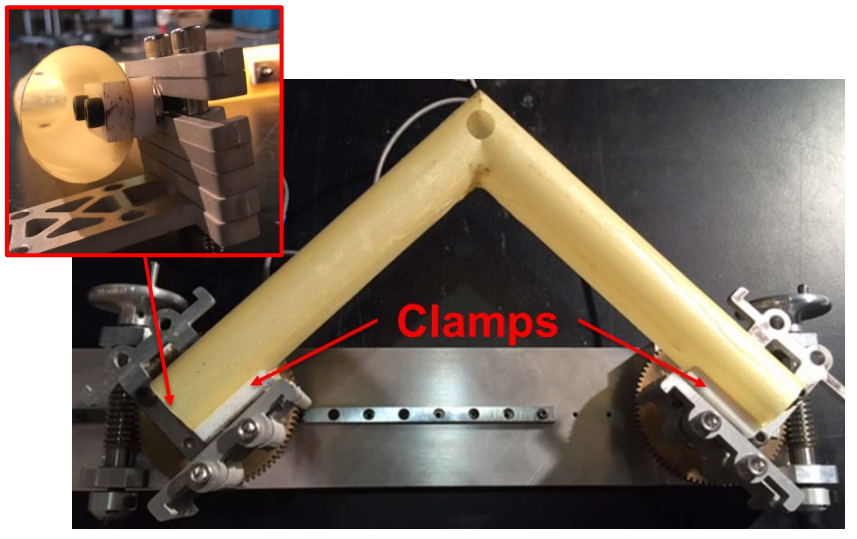

(a)

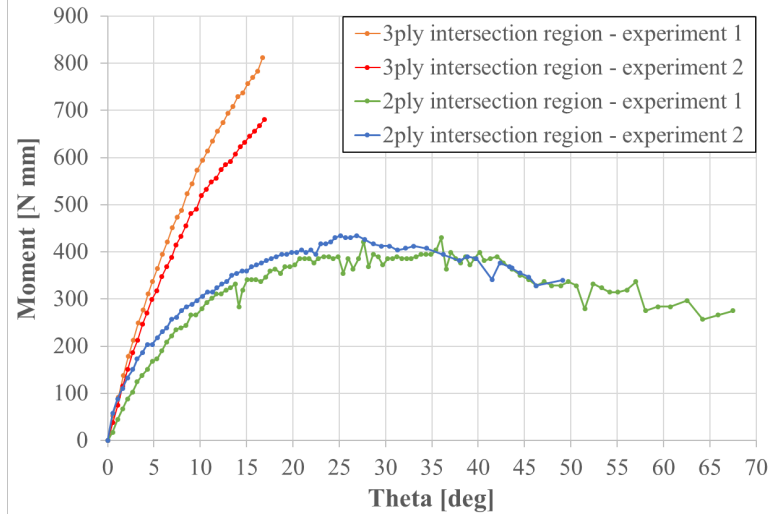

(b)

Figure 4. (a) experimental setup, (b) experimental data of reaction bending moment plotted against the folding angle, moment-angle curves, for second and third iteration joints.

The experimental setup, shown in Figure4(a), consisted of two $35 \mathrm{~mm} \times 15 \mathrm{~mm}$, curved clamps that conformed to the curvature of the cylindrical tubes. The clamps constrained a small region at the end of each tube, thus leaving the end cross-sections free to deform. They also connected the tubes to rotating brackets. The brackets were initially aligned at 45 degrees, to mount the joint. The experiment was rotation controlled. One of the brackets was mounted on ball bearings and it was free to slide towards the other. When the experiment started, the sliding bracket was rotated through less than one degree. The brackets were mounted on gearboxes, connected to strain gauges and a data acquisition system. Once the sliding bracket was rotated, the reaction moment from the tube connected to that bracket could be recorded. The second, fixed bracket was then rotated until the reaction moment recorded on the attached tube equaled the one recorded on the other tube. This process was repeated until the joint was fully folded and both tubes were in contact.

\section{Material Characterization and Failure Criterion}

A laminate failure criterion, ${ }^{10}$ suitable for ultra-thin, plain-weave composites, was used to predict failure of the third iteration joints. The criterion uses three failure indices to capture in-plane, bending, and coupled in-plane and bending failure, defined as follows:

$$
\begin{gathered}
F I_{1}=f_{1}\left(N_{x}+N_{y}\right)+f_{11}\left(N_{x}^{2}+N_{y}^{2}\right)+f_{12} N_{x} N_{y}+f_{33} N_{x y}^{2}<1 \\
F I_{2}=f_{44} \times \max \left(M_{x}^{2}, M_{y}^{2}\right)+f_{66} M_{x y}^{2}<1
\end{gathered}
$$




$$
F I_{3}=\max \left(\frac{N_{x}}{F_{x}}, \frac{N_{y}}{F_{y}}\right)+\frac{\max \left(\left|M_{x}\right|,\left|M_{y}\right|\right)}{F_{4}}<1
$$

Only layups with same-orientation plies can be captured by the criterion. Therefore, only the regions with two and for plies can be analyzed, since all the plies are oriented at \pm 45 degrees. Nevertheless, failure prediction was not compromised because experimental results showed that failure occurs at the joint intersection, where the regions with two and four plies are located. In order to calculate the failure indices for the self-deployable joint, five strength parameters were needed. The tables below summarize the material strength parameters.

Table 1. Material strength parameters for two-ply laminates of Astroquartz fibers and cyanate ester resin.

\begin{tabular}{rrrrr} 
Strength Parameter & 2-Ply Results & Test Samples & Average & Population SD \\
\hline$F_{1 t}=F_{2 t}[\mathrm{~N} / \mathrm{mm}]$ & 71.30 & 5 & 74.89 & 2.83 \\
$F_{1 c}=F_{2 c}[\mathrm{~N} / \mathrm{mm}]$ & 18.19 & - & - & - \\
$F_{3}[\mathrm{~N} / \mathrm{mm}]$ & 4.73 & 5 & 4.95 & 0.27 \\
$F_{4}[\mathrm{~N}]$ & 2.98 & 4 & 3.26 & 0.28 \\
$F_{6}[\mathrm{~N}]$ & 1.00 & 4 & 1.10 & 0.06
\end{tabular}

Table 2. Material strength parameters for four-ply laminates of Astroquartz fibers and cyanate ester resin.

\begin{tabular}{rrrrr} 
Strength Parameter & 4-Ply Results & Test Samples & Average & Population SD \\
\hline$F_{1 t}=F_{2 t}[\mathrm{~N} / \mathrm{mm}]$ & 148.85 & 3 & 150 & 1.47 \\
$F_{1 c}=F_{2 c}[\mathrm{~N} / \mathrm{mm}]$ & 54.87 & - & - & - \\
$F_{3}[\mathrm{~N} / \mathrm{mm}]$ & 14.27 & 5 & 15.44 & 1.07 \\
$F_{4}[\mathrm{~N}]$ & 6.84 & 4 & 8.78 & 1.28 \\
$F_{6}[\mathrm{~N}]$ & 3.49 & 4 & 3.65 & 0.10
\end{tabular}

The tensile strength $F_{1 t}=F_{2 t}$, where 1 and 2 are the directions parallel and perpendicular to the fibers respectively, was measured as the smallest failure value obtained from tensile tests of plain-weave $[0]_{2}$ and $[0]_{4}$ laminates. The compressive strength $F_{1 c}=F_{2 c}$ was calculated using plastic fiber microbuckling criterion. ${ }^{11}$ The basic formulation of this theory is:

$$
\begin{gathered}
F_{1 c}=\frac{G}{1+\frac{\varphi_{0}}{\gamma_{y}}} \\
\gamma_{y}=\frac{\tau_{y}}{G} \\
G=\frac{G_{m}}{1-V_{f}}
\end{gathered}
$$

The fiber misalignment angle, $\varphi_{0}$, was measured from micrographs of samples with one, two, and four plain-weave plies, shown in Figure 5. For a conservative estimate of the compressive strength, the measured angles were not averaged, and only the highest value of 14.9 degrees was used. The in-plane shear strength $F_{3}$ was measured as the smallest failure value obtained from tensile tests of plain-weave $[45]_{2}$ and $[45]_{4}$ laminates, divided by 2 because $N_{x y}=\cos \alpha \sin \alpha N_{x^{\prime}}=0.5 N_{x^{\prime}}$, where $x^{\prime}$ and $y^{\prime}$ are loading directions, $\mathrm{x}$ and $\mathrm{y}$ are fiber directions, and $\alpha=45$ degrees is the fiber orientation angle. The bending strength $F_{4}$ was measured as the smallest failure value obtained from platen bending tests ${ }^{10}$ of plane weave $[0]_{2}$ and $[0]_{4}$ laminates. Finally, the twisting strength $F_{6}$ was measured as the smallest failure value obtained from platen bending tests of plain weave [45] 2 and [45] laminates, divided by 2 because $M_{x y}=\cos \alpha \sin \alpha M_{x^{\prime}}$ $=0.5 M_{x^{\prime}}$. The experiment-based failure criterion was used within the framework of numerical simulations, described in section IV. 

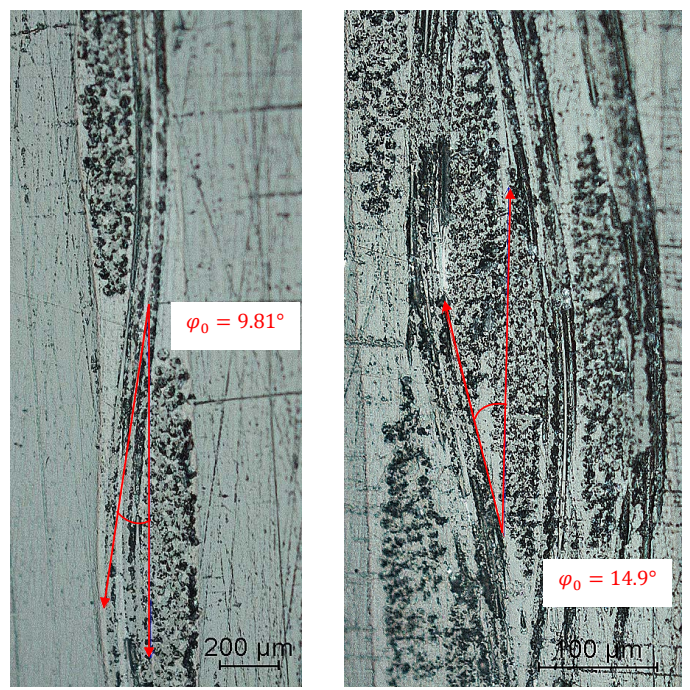

Figure 5. Micrographs of single-ply, [0], and four-ply, [0/0/0/0], plain-weave laminates of Astroquartz and cyanate ester resin.

\section{Numerical Simulations}

In order to characterize the folding behavior of the joints, large-displacement simulations, with linearelastic material, were carried out with finite element software. The model was built in Abaqus 6.14 with S3 shell elements and dynamic/explicit steps. Viscous damping was introduced to obtain quasi-static results. ${ }^{9}$ The four different layups of the third iteration prototype, made of $2,3,4$, and 6 plies, were defined in the model. General section properties were used to match the bending stiffness matrix of each layup, obtained from 4-point bending experiments. To closely replicate the experimental conditions, the simulations were rotation controlled. Mesh convergence studies were performed. The resulting mesh adopted elements with 4 $\mathrm{mm}$ average nodal distance on the tubes, where regions with six and three plies were located, and elements with $1 \mathrm{~mm}$ average nodal distance on the intersection of the joint, where regions with four and two plies were located.

Selected results from the numerical simulations are presented in Figure 6. The contour plot of the inplane strain clearly shows a discontinuity between the tubes and intersection regions where the layup changes. The deformed shape of the joint, observed during the experiments, is well predicted. Sensitivity studies were conducted on both smoothness of the joint between the two cylindrical surfaces and boundary conditions applied at the end cross sections.

It is known that sharp corners favor stress concentration. Additionally, perfectly sharp corners are rarely obtained when building physical models because of manufacturing imperfections. A new type of joint, with smooth intersection, was studied. The radius of curvature of the uppermost contour of the joint between the two cylindrical surfaces, $R_{\text {upper }}$, was designed to be different from the radius of curvature of the lowest contour, $R_{\text {lower }}$. A range between $3 \mathrm{~mm}$ and $14 \mathrm{~mm}$ was assigned to both radii. Figure 6(b) shows the moment-angle curves resulting from the two extreme configurations, blue and orange curves, compared to the joint with perfectly sharp intersection, black curve. The blue curve results from the simulation of a folding joint with $R_{\text {upper }}=14 \mathrm{~mm}$ and $R_{\text {lower }}=3 \mathrm{~mm}$, while the orange curve results from the simulation of a folding joint with $R_{\text {upper }}=3 \mathrm{~mm}$ and $R_{\text {lower }}=14 \mathrm{~mm}$. All the moment-angle curves generated from joints having radii within this range fall between these two curves. One of the most interesting outcomes of this study is the difference in stored strain energy, which corresponds to the area under each curve, of the three configurations. This property could be exploited to tune the strain energy of a folded joint and control the dynamics of its deployment.

The end cross sections of the joint were left free to ovalize during the folding simulations to replicate the boundary conditions of the experimental setup. Only two small regions, $35 \mathrm{~mm} \times 15 \mathrm{~mm}$, were constrained to simulate the effect of the two clamps used during the experiments. The orange moment-angle curve in Figure 6(c) shows the results of these simulations. The end cross section is also shown. Sensitivity studies on 

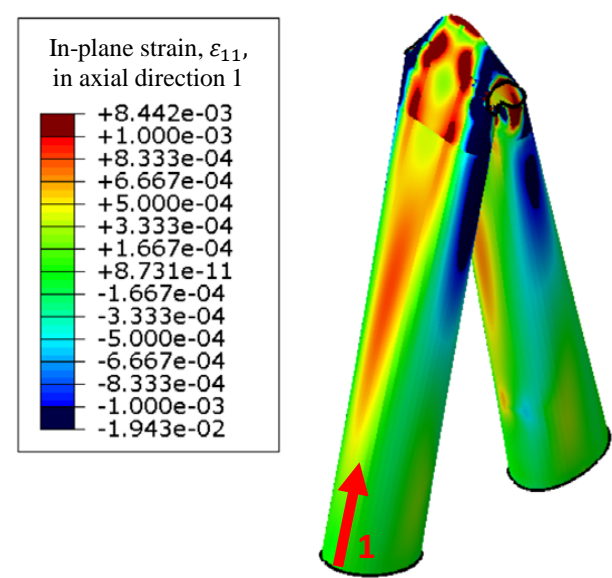

(a)

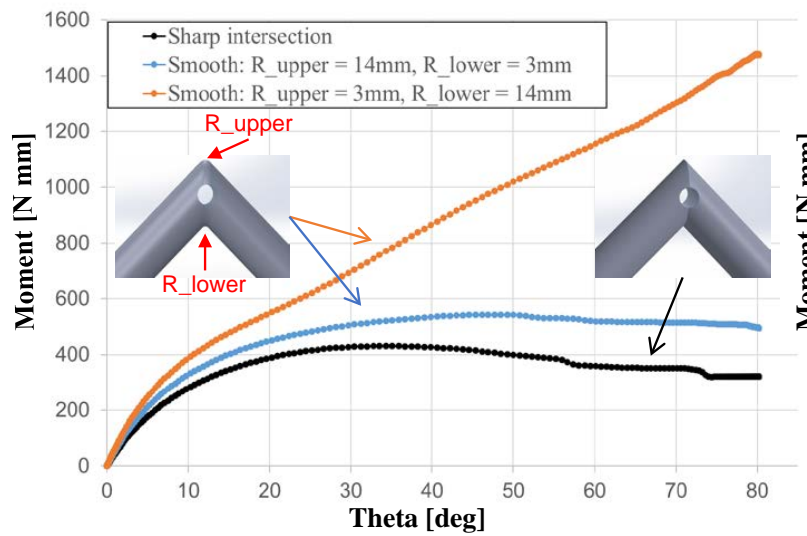

(b)

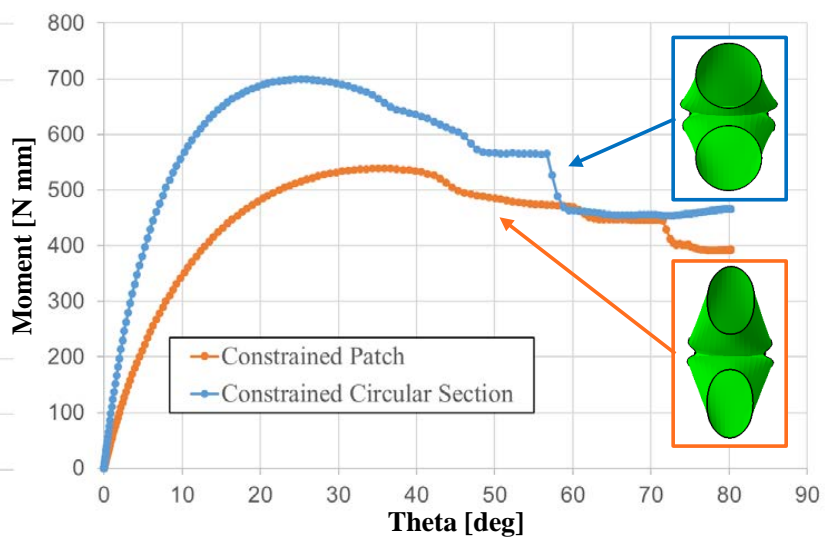

(c)

Figure 6. Results from numerical simulations. (a) contour plot of in-plane strain, $\epsilon_{11}$, along the axial direction of each shaft, (b) sensitivity studies to smoothness of intersection on moment-angle curves, (c) sensitivity studies to boundary conditions on moment-angle curves.

boundary conditions were performed to explore what would happen if the end cross sections were constrained to remain circular. This situation simulates the condition in which the joint is embedded within a larger structure and connected to cylindrical tubes that prevent its end cross sections from deforming freely. As expected, the joint with end cross sections constrained to remain circular shows mostly higher reaction moments for equal folding angles, blue curve in Figure 6(c).

The failure criterion described in III was used to extend the simulations based on linear-elastic material and predict failure of the joints in order to improve their design. The three failure indices were calculated at each step of the simulations and contour plots were obtained. The results of this study showed that the joint fails in the two-ply region and that the first failure index is the most critical, thus suggesting that the self-deployable joint, subjected to bending moments, fails under in-plane stresses. This method produced excellent results, predicting the location of failure and the folding angle at which failure occurs in a conservative manner. Thirty shapes of cutouts, based on intersecting ellipses and rectangles, were investigated. Different radii of curvature for the joint between the two cylindrical surfaces were also explored. Finally, one configuration that limits failure of the joints was found, namely the fourth iteration FRP joint. This particular configuration, with $R_{\text {upper }}=5 \mathrm{~mm}$ and $R_{\text {lower }}=3 \mathrm{~mm}$, is shown in Figure 7 (a). The original model, with circular cutouts and perfectly sharp intersection, and the new joint are compared in terms of in-plane failure index. Only the two-ply regions are plotted while the rest of the joint is left blank. The contour plot covers in black areas with $F I_{1}<1$, hence areas that do not fail. In the new configuration, failure is localized on extremely small areas in the lower part of the joint, thus showing a significant improvement over the original prototype. The failure criterion conservatively used the lowest strength parameters that 

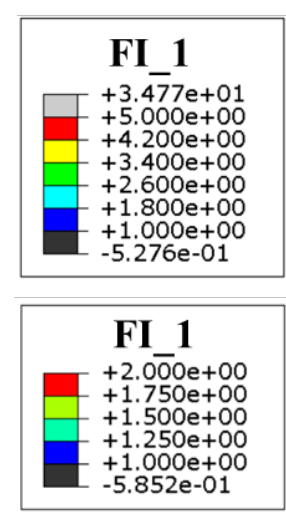
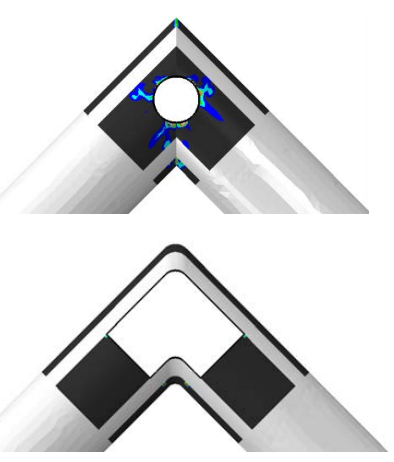

(a)

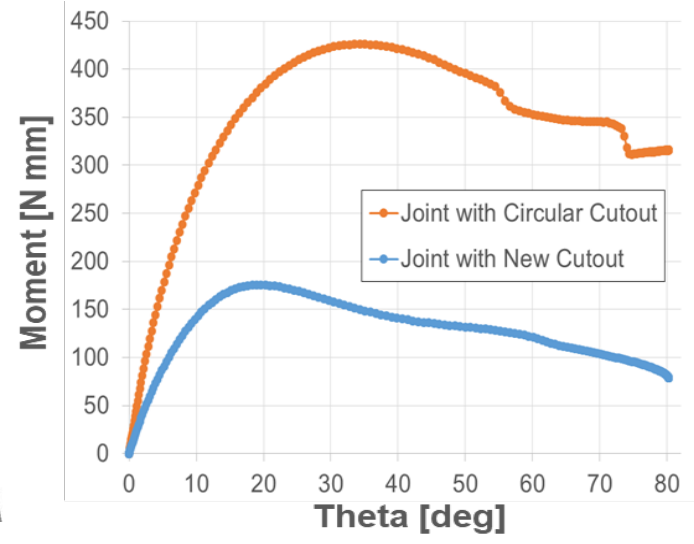

(b)

Figure 7. (a) contour plots of in-plane failure index $F I_{1}$ on latched and folded joints, (b) moment-angle curves for the original joint with circular cutout $(D=14 \mathrm{~mm})$ and the new joint.

were measured during experiments for material characterization. To test the new joint a prototype was manufactured. The results are discussed in section VI. One drawback of the new joint is $50 \%$ bending stiffness reduction of the deployed joint, as shown in the moment-angle plots in Figure 7(b).

\section{Comparison Between Experimental and Numerical Results}

In order to validate the numerical simulations, the moment-angle curve numerically generated for the third iteration joint was plotted against the experimental curves. The results are shown in Figure 8 . The curves closely match up to 25 degrees of folding angle. At this point, the joints have already developed small visible cracks, which are propagating. This behavior cannot be captured in a simulation with linear-elastic material. Hence, the curve numerically generated starts diverging from the experimental curves. During the first folding experiment, green curve, the joint was folded up to 68 degrees. The spikes in the curve capture instabilities due to small cracks, less than $1 \mathrm{~mm}$ long, being formed. These cracks propagate leading to failure of the joint, which in the experimental context is defined as a large crack, more than $5 \mathrm{~mm}$ long, appearing. For the second experiment, blue curve, a new joint was tested up to 50 degrees of folding angle. Stopping the experiment at a lower folding angle prevented small cracks from propagating more than $1 \mathrm{~mm}$, thus avoiding failure of the joint.

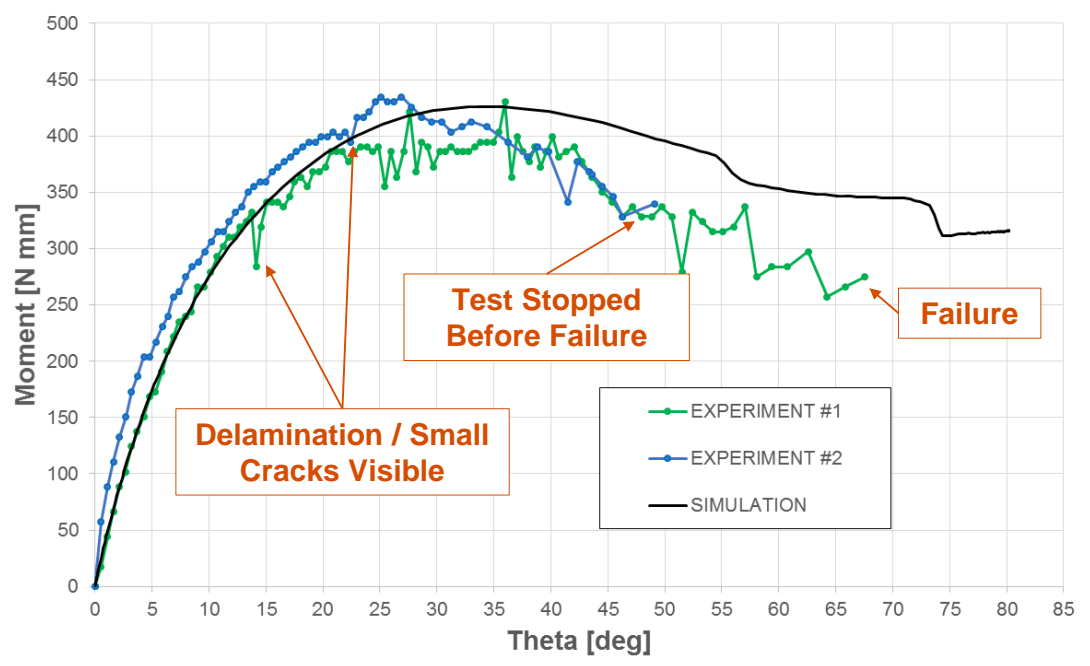

Figure 8. Comparison between numerical simulations of third iteration FRP joint and folding experiments. 


\section{Silicone Molding Manufacturing Technique}

Joints with a sharp intersection were built adopting the simpler manufacturing technique described in section II A. A sharp intersection was easier to build because it imposed no complications on the removal of the internal mold, composed of two separate shafts. In order to build the fourth iteration FRP joints, designed through numerical simulations, a different approach was required. The joint has a smooth intersection, which does not allow an internal mold made of two aluminum shafts.

A novel manufacturing technique was developed, which allowed for faster and cheaper manufacturing processes that can be generalized to different shapes of joints and also adopted to manufacture joints with multiple tubes. The key to this technique was to use silicone rubber to build the internal (male) and external (female) molds needed to support, shape, and apply pressure on the layup. An investigation into different silicone materials resulted in the final use of Mold Max XLS II liquid silicone rubber, from Reynolds Advanced Materials. It has acceptable pour time $(40 \mathrm{~min})$, low mixed viscosity $(30,000 \mathrm{cP})$, and low impact cost on the manufacturing procedure. Silicone rubber thinning fluid, from the same vendor, was also added as $5 \%$ of the total weight to lower the viscosity.
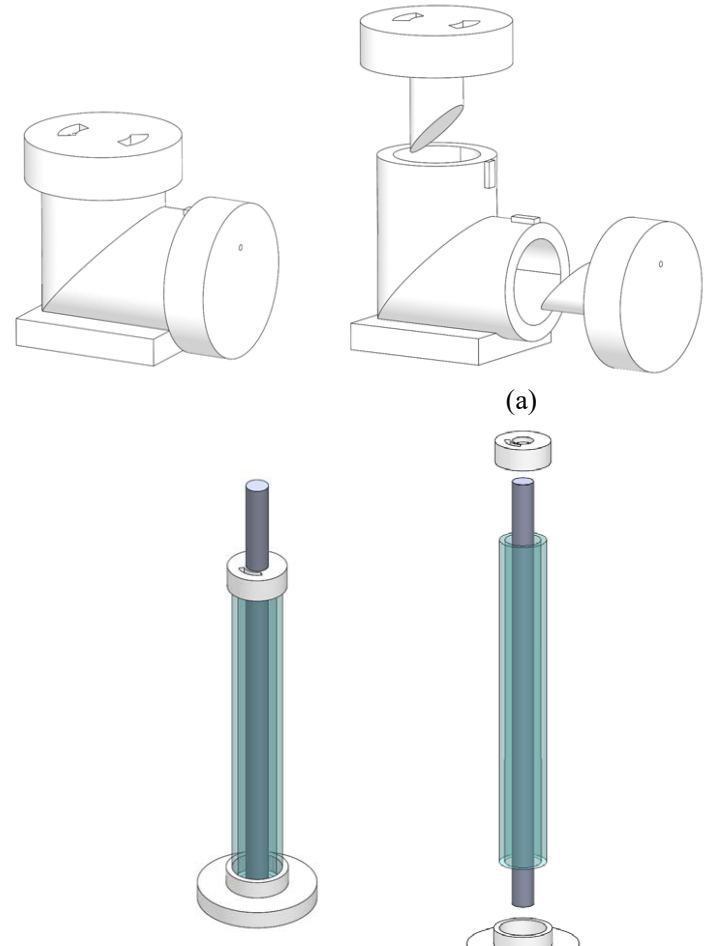

(a)

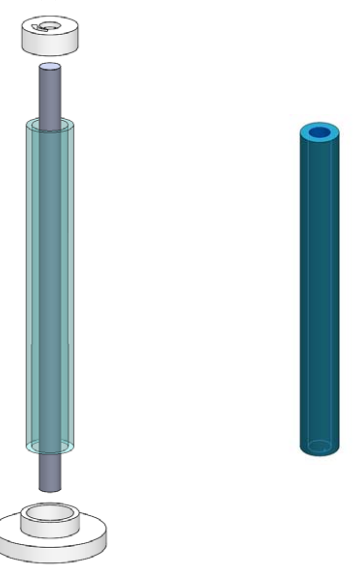

(b)
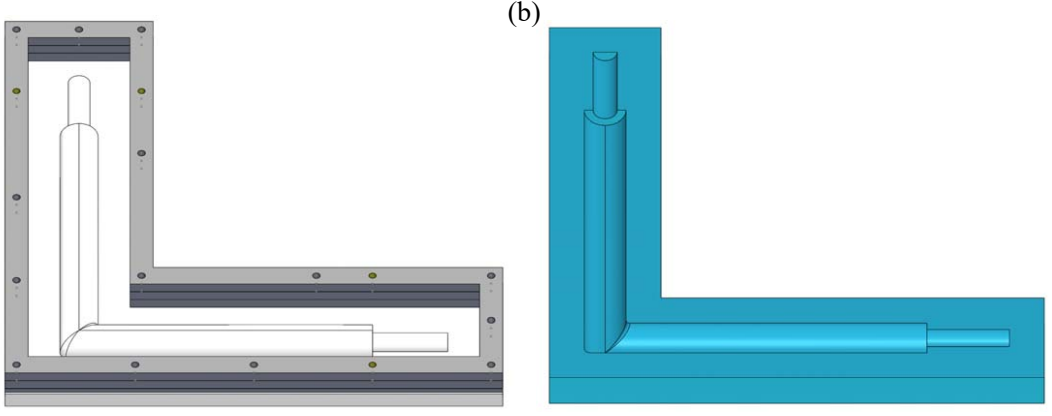

(c)

Figure 9. Design of mandrels used to build male and female silicone mold components. (a) design of L-shaped, 3D printed mandrel used to build intersection component of male silicone mold, (b) assembly of 3D printed caps and commercially available parts used to build two tubes of male silicone mold, (c) assembly of low-density foam base and aluminum cage used to build two halves of female silicone mold. 
The steps involved in manufacturing the silicone molds are shown in Figure 9. The male mold is composed of an intersection piece and two cylindrical tubes. The tree parts are connected through two internal aluminum rods. A 3D printed, L-shaped mandrel is used to build the mold intersection piece. The piece features a smooth joint between the two cylindrical surfaces and two holes that do not connect at the center, thus leaving a small, solid silicone region. The holes host the two thinner aluminum rods, which connect the intersection to the two tubes.

Uncured silicone rubber, with low viscosity, was poured with a syringe through a small, circular cavity placed in one of the caps of the 3D printed mandrel. The silicone was then cured at room temperature for 24 hours. Afterwards, the resulting silicone shape was easily removed from its mandrel. The tubes are hollow cylinders obtained pouring silicone into a mandrel built with 3D printed base and top caps and commercially available materials. The same curing process was applied. The female mold was built of two identical components, which were used to enclose the layup. Due to its size, a low-density foam mandrel, instead of a 3D printed one, and a simple aluminum cage was used to manufacture each component. The whole process was mostly kept low-cost by using inexpensive additive manufacturing techniques and water jetted aluminum parts to build the mandrels. The low-density foam base was manufactured with a computer numerical control (CNC) machining process, which contributed to a cost increase. The silicone rubber molds can be reused multiple times.

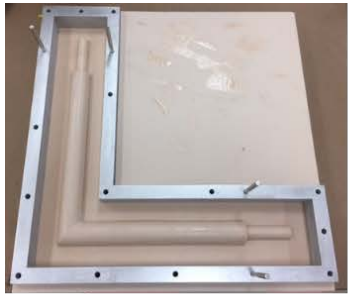

(a)

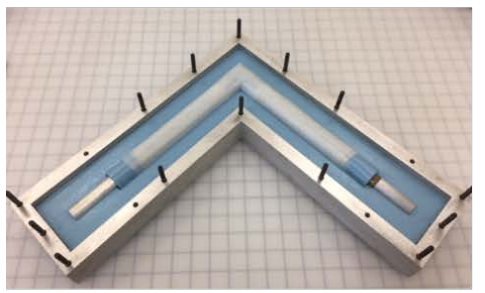

(e)

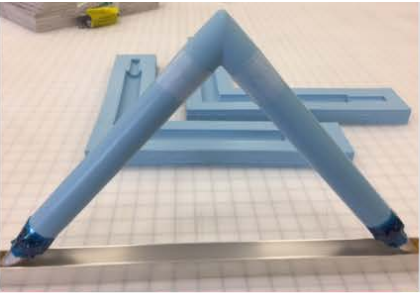

(b)

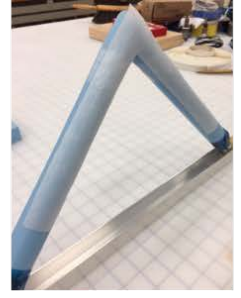

(c)

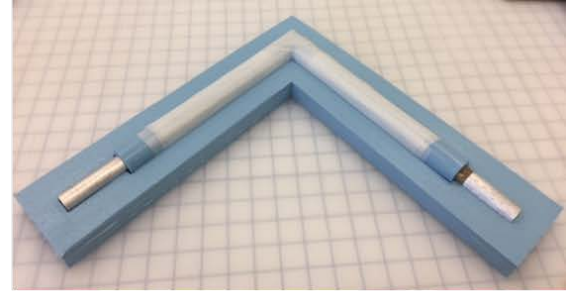

(d)

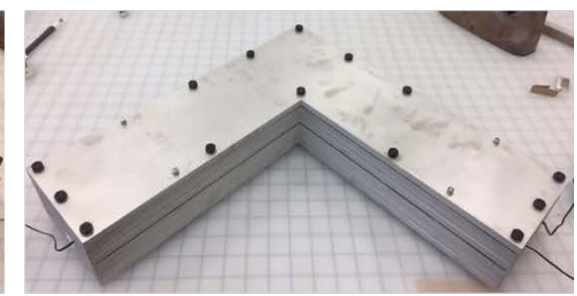

(g)

Figure 10. Manufacturing process with silicone molding. (a) physical model of low-density foam mandrel used to build two halves of female silicone mold, (b) male silicone mold fully assembled and female silicone mold in background, (c) layup process, (d) complete layup positioned inside female silicone mold, (e) aluminum cage used to constrain silicone expansion and provide pressure to cure composite layup, (f) second half of female silicone mold positioned inside the aluminum cage, (g) complete cage.

The layup, shown in Figures 10(c) and 10(d), was assembled directly on the male mold, following the technique described in section II A. The male mold was stiff enough to provide support for assembling the layup. The stiffness comes from the two aluminum rods that run through its core. Once the cure was complete, the aluminum rods could be easily removed and the silicone mold, remaining inside the composite, was compliant enough to be squeezed out. The male mold and the composite layup were positioned within the female mold. An external aluminum cage was used to constrain the expansion of the silicone during the curing process. The high coefficient of thermal expansion of the silicone makes it expand much more compared to the aluminum cage when subjected to high temperatures. This can provide enough pressure for the layup to cure without the need of an autoclave. A high-temperature curing cycle performed in oven can complete the curing process.

Using this technique, one prototype of the fourth iteration FRP joint was built. The material used was 1067 E-glass plain weave fabric, from JPS Composite Materials. This fabric has an areal density of 31 grams per square meter (gsm) instead of the 68 gsm of 525 Astroquartz II. For this reason, the layup was doubled. Regions of two plies became four plies, $[45]_{4}$, four plies became eight, $[45]_{8}$, three plies became six, 
$[45 / 45 / 0]_{s}$, and six plies became twelve, $[45 / 45 / 45 / 45 / 0 / 0]_{s}$. The joint is shown in Figure 11. Preliminary folding experiments showed no signs of failure. The joint was reliably folded and self-deployed multiple times.
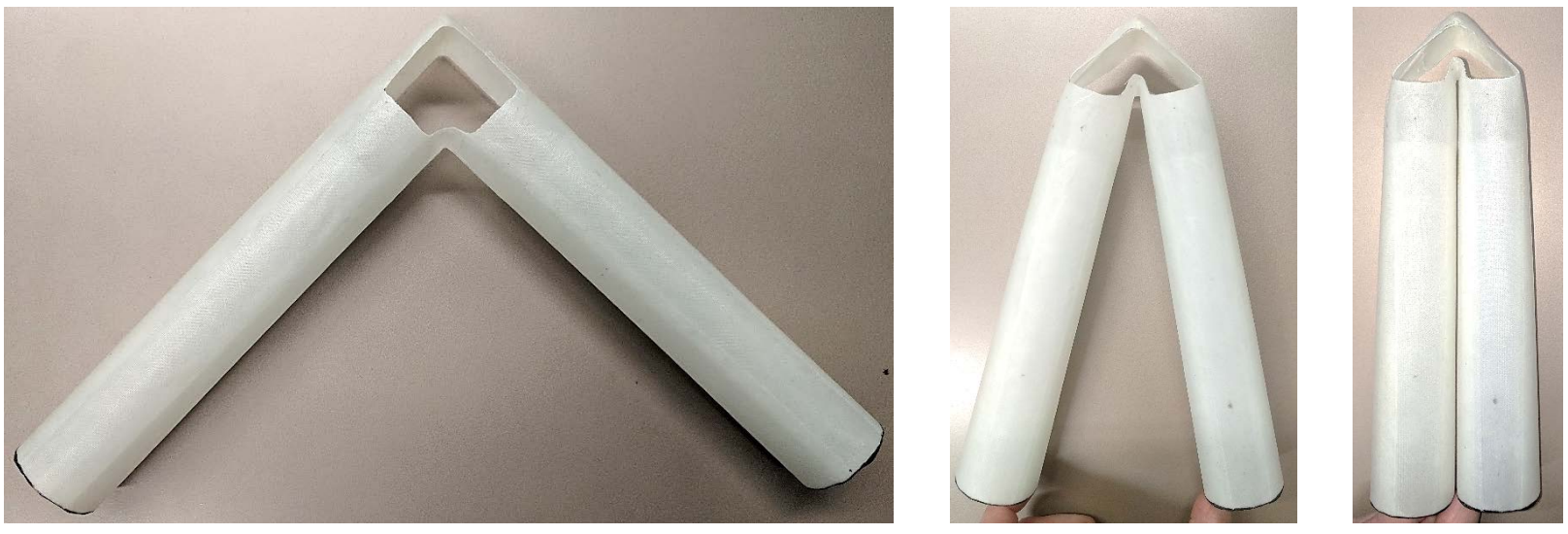

Figure 11. Fourth iteration FRP joint in fully deployed, partially folded, and fully folded configurations.

\section{Conclusion}

Modular, self-deployable structures can be used as building blocks for large space structures. A new way of building these modules, uniquely from composite laminates, is necessary to implement a low-cost and lightweight technology. This introduces the challenge of developing fiber-reinforced-polymers joints, which need to be stiff when deployed, compliant during folding (to avoid failure), and self-deployable.

The paper presented ongoing research on novel concepts for self-latching, flexural joints. The tape-spring hinge idea was explored to build a joint without any mechanisms. Tape-spring hinges are traditionally built in straight tubes. Foldable corner joints that use this idea have not been studied before. The goal of this study was to build a functional corner joint that can fully fold, without failing, and self-deploy.

First, different prototypes of FRP, miter joints were built and the challenges in folding the prototypes were explained through an insight on their deformation modes. The miter joint shape, where two thinwalled cylinders intersect at 90 degrees, was chosen as an initial step towards more complex shapes. A rigid molding manufacturing technique was used to build the first prototypes. The technique allowed to overcome the complications of the non-zero Gaussian curvature at the joint between the two cylindrical surfaces. The results of the manufacturing process were ultra-thin, co-cured tubes, intersected to form a miter joint with a sharp transition between the two cylindrical surfaces.

Next, a detailed characterization of the joints was provided via experiments, a failure criterion, and numerical simulations. A cutout placed at the intersection between the two cylindrical surfaces was found necessary to fold the joint. The reaction moments from the two tubes of each joint were recorded during experiments and plotted against the folding angle. From the moment-angle curves, the stiffness of the second and third iteration FRP joint were measured at $4193.7[\mathrm{~N} \mathrm{~mm}]$ and $1951.9[\mathrm{~N} \mathrm{~mm}]$. Although less stiff, the third iteration FRP joint could be folded up to 50 degrees without failing, compared to the 17 degrees obtained from the second one. Given the improvement obtained with the third iteration joint by changing the layup distribution, material characterization tests were performed to utilize a suitable failure criterion and automate the improvement process. Large-displacement simulations, carried out with finite element software, were validated and used to apply the failure criterion to models of FRP joints featuring different radii of curvature and shapes of the cutout. The results of the simulations lead to a successful design of self-deployable joint.

Finally, an innovative silicone molding manufacturing technique was presented. The manufacturing process was mostly kept low-cost by taking advantage of inexpensive additive manufacturing and machining techniques to build the mandrels for molding the silicone rubber. The silicone rubber molds were used to build the fourth iteration FRP joint, which was successfully and reliably folded and deployed multiple times, without showing signs of failure.

Further research to improve the performance of the joint is currently ongoing. A process for optimizing 
the shape of the cutouts and maximizing the stiffness of the joint, while preventing failure, is being developed. The goal of this research is to build a technique to automatically find the optimal shape and position of the cutouts within the joints. This will expand the results found in this paper to joints with more complex shapes, deployment mechanisms, and out-of-plane degrees of freedom.

\section{Acknowledgments}

This work was supported by a NASA Space Technology Research Fellowship. S.F. would like to acknowledge Dr. William Doggett, NASA Langley, for mentoring and providing invaluable technical and scientific support.

\section{References}

${ }^{1}$ Conley, P.L., Space vehicle mechanisms: elements of successful design, John Wiley \& Sons, 1998.

${ }^{2}$ Pellegrino, S., Kebadze, E., Lefort, T. and Watt, A.M., Low-cost hinge for deployable structures, University of Cambridge, Department of Engineering, 2002.

${ }^{3}$ Footdale, J., Banik, J. and Murphey, T., Design developments of a non-planar deployable structure, In 51st AIAA/ASME/ASCE/AHS/ASC Structures, Structural Dynamics, and Materials Conference 18th AIAA/ASME/AHS Adaptive Structures Conference 12th, 2010.

${ }^{4}$ Francis, W., Lake, M., Mallick, K., Freebury, G. and Maji, A., Development and testing of a hinge/actuator using elastic memory composites, In 44th AIAA/ASME/ASCE/AHS/ASC Structures, Structural Dynamics, and Materials Conference, 2003.

${ }^{5}$ Barrett, R., Francis, W., Abrahamson, E., Lake, M. and Scherbarth, M., Qualification of elastic memory composite hinges for spaceflight applications, In 47th AIAA/ASME/ASCE/AHS/ASC Structures, Structural Dynamics, and Materials Conference 14th AIAA/ASME/AHS Adaptive Structures Conference 7th, 2006.

${ }^{6}$ Hogstrom, K. and Pellegrino, S., Methods for Characterizing the Reliability of Deployable Modules for Large Optical Reflectors, In 3rd AIAA Spacecraft Structures Conference, 2016.

${ }^{7}$ Lee, N., Backes, P., Burdick, J., Pellegrino, S., Fuller, C., Hogstrom, K., Kennedy, B., Kim, J., Mukherjee, R., Seubert, C. and Wu, Y.H., Architecture for in-space robotic assembly of a modular space telescope, Journal of Astronomical Telescopes, Instruments, and Systems, 2(4), pp.041207-041207, 2016.

${ }^{8}$ Polidan, R.S., Breckinridge, J.B., Lillie, C.F., MacEwen, H.A., Flannery, M.R. and Dailey, D.R., An evolvable space telescope for future astronomical missions, In SPIE Astronomical Telescopes+ Instrumentation (pp. 914319-914319). International Society for Optics and Photonics, 2014.

${ }^{9}$ Mallikarachchi, H.M.Y.C. and Pellegrino, S., Quasi-static folding and deployment of ultrathin composite tape-spring hinges, Journal of Spacecraft and Rockets, 48(1), pp.187-198, 2011.

${ }^{10}$ Mallikarachchi, H.M.Y.C. and Pellegrino, S., Failure criterion for two-ply plain-weave CFRP laminates, Journal of Composite Materials, 47(11), pp.1357-1375, 2013.

${ }^{11}$ Fleck, N.A. and Budiansky, B., Compressive failure of fibre composites due to microbuckling, In Inelastic deformation of composite materials (pp. 235-273). Springer New York, 1991. 